\title{
RATE OF APPROXIMATION FOR MULTIVARIATE SAMPLING KANTOROVICH OPERATORS ON SOME FUNCTIONS SPACES
}

\author{
DANILO COSTARELLI AND GIANLUCA VINTI
}

\begin{abstract}
In this paper, the problem of the order of approximation for the multivariate sampling Kantorovich operators is studied. The cases of uniform approximation for uniformly continuous and bounded functions/signals belonging to Lipschitz classes and the case of the modular approximation for functions in Orlicz spaces are considered. In the latter context, Lipschitz classes of Zygmund-type which take into account of the modular functional involved are introduced. Applications to $L^{p}\left(\mathbb{R}^{n}\right)$, interpolation and exponential spaces can be deduced from the general theory formulated in the setting of Orlicz spaces. The special cases of multivariate sampling Kantorovich operators based on kernels of the product type and constructed by means of Fejér's and B-spline kernels have been studied in details.
\end{abstract}

1. Introduction. The sampling Kantorovich operators have been introduced to approximate and reconstruct not necessarily continuous signals. The multivariate sampling Kantorovich operators considered in this paper $([\mathbf{2 7}])$ are of the form:

$$
\left(S_{w} f\right)(\underline{x}):=\sum_{\underline{k} \in \mathbb{Z}^{n}} \chi\left(w \underline{x}-t_{\underline{k}}\right)\left[\frac{w^{n}}{A_{\underline{k}}} \int_{R_{\underline{k}}^{w}} f(\underline{u}) d \underline{u}\right], \quad\left(\underline{x} \in \mathbb{R}^{n}\right),
$$

where $f: \mathbb{R}^{n} \rightarrow \mathbb{R}$ is a locally integrable function such that the above series is convergent for every $\underline{x} \in \mathbb{R}^{n}$. The symbol $t_{k}=\left(t_{k_{1}}, \ldots, t_{k_{n}}\right)$ denotes vectors where each $\left(t_{k_{i}}\right)_{k_{i} \in \mathbb{Z}}, i=1, \ldots, n$ is a certain strictly increasing sequence of real numbers with $\Delta_{k_{i}}=t_{k_{i+1}}-t_{k_{i}}>0$. Note that the sequences $\left(t_{k_{i}}\right)_{k_{i} \in \mathbb{Z}}$ are not necessary equally spaced (irregular

2010 AMS Mathematics subject classification. Primary 41A25, 41A30, 46E30, 47A58, 47B38, 94A12.

Keywords and phrases. Multivariate sampling Kantorovich operators, Orlicz spaces, order of approximation, Lipschitz classes, irregular sampling.

The first author is the corresponding author.

Received by the editors on June 9, 2014. 
sampling scheme). We denote by $R_{\underline{k}}^{w}$ the sets:

$R_{\underline{k}}^{w}:=\left[\frac{t_{k_{1}}}{w}, \frac{t_{k_{1}+1}}{w}\right] \times\left[\frac{t_{k_{2}}}{w}, \frac{t_{k_{2}+1}}{w}\right] \times \cdots \times\left[\frac{t_{k_{n}}}{w}, \frac{t_{k_{n}+1}}{w}\right]$,

$w>0$ and $A_{\underline{k}}=\Delta_{k_{1}} \cdot \Delta_{k_{2}} \cdot \ldots \cdot \Delta_{k_{n}}, \underline{k} \in \mathbb{Z}^{n}$. Moreover, the function

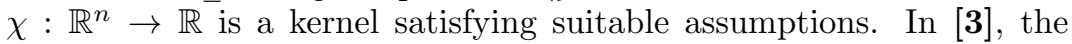
authors introduced these operators in the univariate form, starting from the well-known generalized sampling operators (see e.g., $[\mathbf{2}, \mathbf{4}$, $12,14,16,45,9,46,47,10,43])$ and replacing, in their definition, the sample values $f(k / w)$ with $w \int_{k / w}^{(k+1) / w} f(u) d u$. Clearly, this is the most natural mathematical modification to obtain operators which can be well-defined also for general measurable, locally integrable functions, not necessarily continuous. Moreover, this situation very often occurs in Signal Processing, when one cannot match exactly the sample at the point $k / w$ : this represents the so-called "time-jitter" error. The theory of sampling Kantorovich operators allows us to reduce the time-jitter error, calculating the information in a neighborhood of $k / w$ rather than exactly in the node $k / w$. These operators, as the generalized sampling operators, represent an approximate version of the classical sampling series, based on the Whittaker-Kotelnikov-Shannon sampling theorem (see e.g., $[1,11,15,35,31,33,34]$ ).

Subsequently, the sampling Kantorovich operators have been studied in various settings. In $[\mathbf{2 8}, \mathbf{4 8}]$ the nonlinear univariate and multivariate versions of these operators were introduced; applications to image processing have been discussed in $[\mathbf{2 7}, \mathbf{2 8}]$. Indeed, static gray scale images are characterized by jumps of gray levels mainly concentrated in their contours or edges, and this can be translated, from a mathematical point of view, by discontinuities. For these reasons, multivariate sampling Kantorovich operators appear very appropriate for applications to image reconstruction. Moreover, some preliminary applications to civil engineering have been presented in $[\mathbf{1 7}, \mathbf{1 8}, \mathbf{1 9}]$. Results concerning the order of approximation have been obtained in $[\mathbf{2 9}, \mathbf{3 0}]$ in the univariate case, for the linear and nonlinear versions of these operators. Extensions of the theory to more general contexts were presented in $[6,32,44,49,50]$.

In this paper, we study the problem of the rate of approximation for the multivariate sampling Kantorovich operators in various settings. More precisely, we consider the case of uniform approximation 
for uniformly continuous and bounded functions belonging to Lipschitz classes and the case of the modular approximation for functions in Orlicz spaces $L^{\varphi}\left(\mathbb{R}^{n}\right)$. In this context, we will introduce Lipschitz classes of Zygmund-type which take into account the modular functional involved. From the results concerning Orlicz spaces, applications to $L^{p}\left(\mathbb{R}^{n}\right)$ spaces, $1 \leq p<+\infty$, as to other examples of Orlicz spaces can be deduced. In particular, the application of the multivariate sampling Kantorovich operators to $L^{p}$-spaces is suitable for Signal/Image Processing. Other important cases of Orlicz spaces considered here are the interpolation spaces $L^{\alpha} \log ^{\beta} L\left(\mathbb{R}^{n}\right)$ and the exponential spaces, which are very useful for applications, e.g., to partial differential equations and for embedding theorems between Sobolev spaces, respectively.

In order to obtain results concerning the order of approximation for the multivariate sampling Kantorovich operators starting from the one-dimensional theory, some difficulties arise. First of all, the definition of the Lipschitz class in which we work must be extended to the multivariate case, both in the case of uniformly continuous functions and for functions in Orlicz spaces. But the main difficulty is related to the construction of multivariate kernels which satisfy all the assumptions of the above theory (see Section 6). Indeed, it turns out that the kernels affect the rate of approximations when uniform and modular approximation are considered. For this reason, we introduce a procedure useful to construct multivariate kernels and to determine their parameters $\mu, \beta$ and $\alpha$, starting from one-dimensional kernels. The special cases of multivariate kernels of the product type, based upon Fejér's and B-spline kernels have been studied in detail.

2. Preliminary notions. In this paper, we will denote by $C\left(\mathbb{R}^{n}\right)$ the set of all uniformly continuous and bounded functions $f: \mathbb{R}^{n} \rightarrow \mathbb{R}$ endowed with the usual sup-norm $\|\cdot\|_{\infty}$. Moreover, by $\|\cdot\|_{2}$, we will denote the usual Euclidean norm in $\mathbb{R}^{n}$, i.e., $\|\underline{x}\|_{2}:=\left(x_{1}^{2}+\cdots+x_{n}^{2}\right)^{1 / 2}$, $\underline{x}=\left(x_{1}, \ldots, x_{n}\right) \in \mathbb{R}^{n}$.

In order to study the rate of approximation of a family of linear operators, we introduce the definition of the Zygmund-type class (Lipschitz class) for functions of several variables. 
We define the class $\operatorname{Lip}_{\infty}(\nu), 0<\nu \leq 1$, as

$$
\begin{aligned}
\operatorname{Lip}_{\infty}(\nu) & :=\left\{f \in C\left(\mathbb{R}^{n}\right):\|f(\cdot)-f(\cdot+\underline{t})\|_{\infty}\right. \\
& \left.=\mathcal{O}\left(\|\underline{t}\|_{2}^{\nu}\right), \text { as }\|\underline{t}\|_{2} \rightarrow 0\right\},
\end{aligned}
$$

where for any two functions $f, g: \mathbb{R}^{n} \rightarrow \mathbb{R}, f(\underline{t})=\mathcal{O}(g(\underline{t}))$ as $\|\underline{t}\|_{2} \rightarrow 0$ means that there exist constants $C, \gamma>0$ such that $|f(\underline{t})| \leq C|g(\underline{t})|$ for every $\underline{t} \in \mathbb{R}^{n}$, with $\|\underline{t}\|_{2} \leq \gamma([7,47])$. The above definition represents the extension to the multivariate setting of the Zygmundtype classes introduced in [29] for univariate functions. It is easy to observe that, in the case of functions defined on bounded domains, the above definition is equivalent to the well-known definition of $\nu$-Holder continuous functions.

The sampling Kantorovich operators $S_{w}$ studied in this paper are suitable to reconstruct not necessarily continuous signals (see, e.g., $[3,27]$ ), and a very general class of spaces containing such functions are the so-called Orlicz spaces. Since in the rest of the paper we will study the above operators also in this general setting, we now recall some basic notions concerning Orlicz spaces.

A function $\varphi: \mathbb{R}_{0}^{+} \rightarrow \mathbb{R}_{0}^{+}$is said to be a $\varphi$-function if it satisfies the following conditions:

$(\Phi 1) \varphi$ is a non decreasing and continuous function;

$(\Phi 2) \varphi(0)=0, \varphi(u)>0$ if $u>0$ and $\lim _{u \rightarrow+\infty} \varphi(u)=+\infty$.

Let us now consider the functional $I^{\varphi}$ associated to any given $\varphi$ function $\varphi$ and defined as follows

$$
I^{\varphi}[f]:=\int_{\mathbb{R}^{n}} \varphi(|f(\underline{x})|) d \underline{x},
$$

for every $f \in M\left(\mathbb{R}^{n}\right)$, i.e., for every (Lebesgue) measurable function $f: \mathbb{R}^{n} \rightarrow \mathbb{R}$. As it is well-known, the functional $I^{\varphi}$ satisfies a number of important properties. For instance, $I^{\varphi}$ is a modular functional (see e.g., $[7,39,41])$, and moreover, if the $\varphi$-function $\varphi$ is convex, the corresponding modular functional is convex too.

Now, we are able to recall the definition of the Orlicz space $L^{\varphi}\left(\mathbb{R}^{n}\right)$ generated by $\varphi$. We define

$$
L^{\varphi}\left(\mathbb{R}^{n}\right):=\left\{f \in M\left(\mathbb{R}^{n}\right): I^{\varphi}[\lambda f]<+\infty \text {, for some } \lambda>0\right\} .
$$

A notion of convergence in Orlicz spaces, called modular convergence, 
was introduced in [40], which induces a topology in $L^{\varphi}\left(\mathbb{R}^{n}\right)$, called modular topology.

A family $\left(f_{w}\right)_{w>0} \subset L^{\varphi}\left(\mathbb{R}^{n}\right)$ is said to be modularly convergent to $f \in L^{\varphi}\left(\mathbb{R}^{n}\right)$, if there exists $\lambda>0$ such that

$$
\begin{gathered}
I^{\varphi}\left[\lambda\left(f_{w}-f\right)\right]=\int_{\mathbb{R}^{n}} \varphi\left(\lambda\left|f_{w}(\underline{x})-f(\underline{x})\right|\right) d \underline{x} \longrightarrow 0, \\
\text { as } w \rightarrow+\infty .
\end{gathered}
$$

Moreover we recall, for the sake of completeness, that in $L^{\varphi}\left(\mathbb{R}^{n}\right)$ can be also given a stronger notion of convergence, i.e., the Luxemburg-norm convergence, see e.g. [7, 39].

We now define by $\operatorname{Lip}_{\varphi}(\nu), 0<\nu \leq 1$, the Zygmung-type class in Orlicz spaces, as the set of all functions $f \in M\left(\mathbb{R}^{n}\right)$ such that there exists $\lambda>0$ with

$$
I^{\varphi}[\lambda(f(\cdot)-f(\cdot+\underline{t}))]=\int_{\mathbb{R}^{n}} \varphi(\lambda|f(\underline{x})-f(\underline{x}+\underline{t})|) d \underline{x}=\mathcal{O}\left(\|\underline{t}\|_{2}^{\nu}\right),
$$

as $\|\underline{t}\|_{2} \rightarrow 0$. The above definition extends that given in [29] for functions of one variable. For further results concerning Orlicz spaces, see $[5,7,8,36,37,38,39,41,42]$.

3. Multivariate sampling Kantorovich operators. In this section, the definition of the multivariate sampling Kantorovich operators is recalled $([\mathbf{2 7}])$, together with its main properties and some useful remarks.

Let $\Pi^{n}=\left(t_{k}\right)_{k \in \mathbb{Z}^{n}}$ be a sequence of real numbers defined by $t_{\underline{k}}=\left(t_{k_{1}}, \ldots, t_{k_{n}}\right)$, where each $\left(t_{k_{i}}\right)_{k_{i} \in \mathbb{Z}}, i=1, \ldots, n$, is a sequence such that $-\infty<t_{k_{i}}<t_{k_{i}+1}<+\infty$ for every $k_{i} \in \mathbb{Z}, \lim _{k_{i} \rightarrow \pm \infty} t_{k_{i}}= \pm \infty$, and there are two positive constants $\Delta, \delta$ such that $\delta \leq \Delta_{k_{i}}:=$ $t_{k_{i}+1}-t_{k_{i}} \leq \Delta$, for every $k_{i} \in \mathbb{Z}$.

In what follows, a function $\chi: \mathbb{R}^{n} \rightarrow \mathbb{R}$ will be called a kernel if it satisfies the following properties:

$(\chi 1) \chi \in L^{1}\left(\mathbb{R}^{n}\right)$ and is bounded in a neighborhood of the origin;

$(\chi 2)$ For some $\mu>0$,

$$
\sum_{\underline{k} \in \mathbb{Z}^{n}} \chi\left(w \underline{x}-t_{\underline{k}}\right)-1=: A_{w}(\underline{x})-1=\mathcal{O}\left(w^{-\mu}\right), \quad \text { as } w \rightarrow+\infty,
$$


uniformly with respect to $\underline{x} \in \mathbb{R}^{n}$;

$(\chi 3)$ For some $\beta>0$, we assume that the discrete absolute moment of order $\beta$ is finite, i.e.,

$$
m_{\beta, \Pi^{n}}(\chi):=\sup _{\underline{u} \in \mathbb{R}} \sum_{\underline{k} \in \mathbb{Z}^{n}}\left|\chi\left(\underline{u}-t_{\underline{k}}\right)\right| \cdot\left\|\underline{u}-t_{\underline{k}}\right\|_{2}^{\beta}<+\infty ;
$$

( $\chi 4)$ There exists $\alpha>0$ such that, for every $M>0$,

$$
\int_{\|\underline{u}\|_{2}>M} w^{n}|\chi(w \underline{u})| d \underline{u}=\mathcal{O}\left(w^{-\alpha}\right), \quad \text { as } w \rightarrow+\infty .
$$

The conditions listed above are the typical properties satisfied by the approximate identities and are standard assumptions required in the case of discrete linear operators.

In order to recall the definition of sampling Kantorovich operators, we first introduce the following notation:

$$
R_{\underline{k}}^{w}:=\left[\frac{t_{k_{1}}}{w}, \frac{t_{k_{1}+1}}{w}\right] \times \cdots \times\left[\frac{t_{k_{n}}}{w}, \frac{t_{k_{n}+1}}{w}\right] \subset \mathbb{R}^{n},
$$

for every $\underline{k} \in \mathbb{Z}^{n}$ and $w>0$. Denoting by $A_{k}:=\Delta_{k_{1}} \cdot \ldots \cdot \Delta_{k_{n}}$, the Lebesgue measure of $R_{\underline{k}}^{w}$ is given by $A_{\underline{k}} / w^{n}$. We now define by $\left(S_{w}\right)_{w>0}$ the family of the multivariate sampling Kantorovich operators defined by

$$
\left(S_{w} f\right)(\underline{x}):=\sum_{\underline{k} \in \mathbb{Z}^{n}} \chi\left(w \underline{x}-t_{\underline{k}}\right)\left[\frac{w^{n}}{A_{\underline{k}}} \int_{R_{\underline{k}}^{w}} f(\underline{u}) d \underline{u}\right] \quad\left(\underline{x} \in \mathbb{R}^{n}\right),
$$

where $f: \mathbb{R}^{n} \rightarrow \mathbb{R}$ is a locally integrable function such that the series is convergent for every $\underline{x} \in \mathbb{R}^{n}$.

We begin giving the proof of following lemma.

Lemma 3.1. Under the assumptions $(\chi 1)$ and $(\chi 3)$ on the kernel $\chi$, we have:

(i) $m_{0, \Pi^{n}}(\chi):=\sup _{\underline{u} \in \mathbb{R}^{n}} \sum_{\underline{k} \in \mathbb{Z}^{n}}\left|\chi\left(\underline{u}-t_{\underline{k}}\right)\right|<+\infty$;

(ii) For every $\gamma>0$,

$$
\sum_{\left\|w \underline{x}-t_{\underline{t}}\right\|_{2}>\gamma w}\left|\chi\left(w \underline{x}-t_{\underline{k}}\right)\right|=\mathcal{O}\left(w^{-\beta}\right), \quad \text { as } w \rightarrow+\infty,
$$


uniformly with respect to $\underline{x} \in \mathbb{R}^{n}$, where $\beta>0$ is the constant of condition $(\chi 3)$.

Proof. For a proof of (i), see e.g. [27].

(ii) Let $\gamma>0$ be fixed. For every $\underline{x} \in \mathbb{R}^{n}$ and $w>0$, we obtain

$$
\begin{aligned}
\sum_{\left\|w \underline{x}-t_{\underline{k}}\right\|_{2}>\gamma w}\left|\chi\left(w \underline{x}-t_{\underline{k}}\right)\right| & \leq \frac{1}{\gamma^{\beta} w^{\beta}} \sum_{\left\|w \underline{x}-t_{\underline{k}}\right\|_{2}>\gamma w}\left|\chi\left(w \underline{x}-t_{\underline{k}}\right)\right|\left\|w \underline{x}-t_{\underline{k}}\right\|_{2}^{\beta} \\
& \leq \frac{1}{\gamma^{\beta} w^{\beta}} m_{\beta, \Pi^{n}}(\chi)<+\infty
\end{aligned}
$$

and so the assertion follows.

Remark 3.2. In the case of $f \in L^{\infty}\left(\mathbb{R}^{n}\right)$, by Lemma 3.1 (i), $S_{w} f$ are well-defined for every $w>0$. Indeed,

$$
\left|\left(S_{w} f\right)(\underline{x})\right| \leq m_{0, \Pi^{n}}(\chi)\|f\|_{\infty}<+\infty,
$$

for every $\underline{x} \in \mathbb{R}^{n}$ and $w>0$, i.e., $S_{w}: L^{\infty}\left(\mathbb{R}^{n}\right) \rightarrow L^{\infty}\left(\mathbb{R}^{n}\right)$.

4. Order of approximation in $C\left(\mathbb{R}^{n}\right)$. We now begin by studying the rate of approximation for the family of linear, multivariate sampling Kantorovich operators (2) in $C\left(\mathbb{R}^{n}\right)$.

Theorem 4.1. Let $\chi$ be a kernel and $f \in \operatorname{Lip}_{\infty}(\nu), 0<\nu \leq 1$. Then

$$
\left\|S_{w} f-f\right\|_{\infty}=\mathcal{O}\left(w^{-\epsilon}\right), \quad \text { as } w \rightarrow+\infty
$$

where $\epsilon:=\min \{\nu, \beta, \mu\}$ and $\mu, \beta>0$ are the constants of conditions $(\chi 2)$ and $(\chi 3)$, respectively.

Proof. First, we consider the case of $\chi$ satisfying condition $(\chi 3)$ for $0<\beta \leq 1$.

Now let $f \in \operatorname{Lip}_{\infty}(\nu), 0<\nu \leq \beta$, be fixed. By Remark 3.2, $S_{w} f$ are well defined for every $w>0$. Moreover, since $f \in \operatorname{Lip}_{\infty}(\nu)$, we have

$$
\sup _{\underline{x} \in \mathbb{R}^{n}}|f(\underline{x})-f(\underline{x}+\underline{t})| \leq C\|\underline{t}\|_{2}^{\nu}
$$


for some constants $C, \gamma>0$, and for every $\underline{t} \in \mathbb{R}^{n}$ such that $\|\underline{t}\|_{2} \leq \gamma$. Now let $\underline{x} \in \mathbb{R}^{n}$ be fixed. Then we can write

$$
\begin{aligned}
\left|\left(S_{w} f\right)(\underline{x})-f(\underline{x})\right| \leq & \left|\left(S_{w} f\right)(\underline{x})-f(\underline{x}) A_{w}(\underline{x})\right| \\
& +\left|f(\underline{x}) A_{w}(\underline{x})-f(\underline{x})\right| \\
\leq & \sum_{\underline{k} \in \mathbb{Z}^{n}}\left|\chi\left(w \underline{x}-t_{\underline{k}}\right)\right| \frac{w^{n}}{A_{\underline{k}}} \int_{R_{\underline{k}}^{w}}|f(\underline{u})-f(\underline{x})| d \underline{u} \\
& +|f(\underline{x})|\left|A_{w}(\underline{x})-1\right| \\
\leq & \left(\sum_{\left\|w \underline{x}-t_{\underline{k}}\right\|_{2} \leq w \gamma / 2}+\sum_{\underline{w}-t_{\underline{k}} \|_{2}>w \gamma / 2}\right) \\
& \left|\chi\left(w \underline{x}-t_{\underline{k}}\right)\right| \frac{w^{n}}{A_{\underline{k}}} \int_{R_{\underline{k}}^{w}}|f(\underline{u})-f(\underline{x})| d \underline{u} \\
& +\|f\|_{\infty}\left|A_{w}(\underline{x})-1\right|=: I_{1}+I_{2}+I_{3} .
\end{aligned}
$$

In order to estimate $I_{1}$, we now introduce the following notation. We denote by $\left(R_{\underline{k}}^{w}-\underline{x}\right)$ sets of the form

$$
\left(R_{\underline{k}}^{w}-\underline{x}\right):=\left[\frac{t_{k_{1}}}{w}-x_{1}, \frac{t_{k_{1}+1}}{w}-x_{1}\right] \times \cdots \times\left[\frac{t_{k_{n}}}{w}-x_{n}, \frac{t_{k_{n}+1}}{w}-x_{n}\right] .
$$

We can observe that, for every $\underline{t} \in\left(R_{\underline{k}}^{w}-\underline{x}\right)$, if $\left\|w \underline{x}-t_{\underline{k}}\right\|_{2} \leq w \gamma / 2$, we have

$$
\|\underline{t}\|_{2} \leq\left\|\underline{t}-t_{\underline{k}} / w+\underline{x}\right\|_{2}+\left\|t_{\underline{k}} / w-\underline{x}\right\|_{2} \leq \sqrt{n} \frac{\Delta}{w}+\frac{\gamma}{2}<\gamma,
$$

for sufficiently large $w>0$. Then, by the change of variable $\underline{u}=\underline{x}+\underline{t}$ in the integrals of $I_{1}$, the above inequality and the definition of $\operatorname{Lip}_{\infty}(\nu)$, we can obtain

$$
\begin{aligned}
I_{1}= & \sum_{\left\|w \underline{x}-t_{\underline{k}}\right\|_{2} \leq w \gamma / 2}\left|\chi\left(w \underline{x}-t_{\underline{k}}\right)\right| \frac{w^{n}}{A_{\underline{k}}} \\
& \times \int_{\left(R_{\underline{k}}^{w}-\underline{x}\right)}|f(\underline{x}+\underline{t})-f(\underline{x})| d \underline{t} \\
\leq & C \sum_{\left\|w \underline{x}-t_{\underline{k}}\right\|_{2} \leq w \gamma / 2}\left|\chi\left(w \underline{x}-t_{\underline{k}}\right)\right| \frac{w^{n}}{A_{\underline{k}}} \int_{\left(R_{\underline{k}}^{w}-\underline{x}\right)}\|\underline{t}\|_{2}^{\nu} d \underline{t} .
\end{aligned}
$$


In order to estimate (3), we proceed as follows:

$$
\begin{aligned}
\max _{\underline{t} \in\left(R_{\underline{w}}^{w}-\underline{x}\right)}\|\underline{t}\|_{2}^{\nu} \leq & \left.\max \max _{t_{1} \in\left[\frac{t_{k_{1}}}{w}-x_{1}, \frac{t_{k_{1}+1}}{w}-x_{1}\right]} t_{1}^{2}+\cdots+\tan _{n} \in\left[\frac{t_{k_{n}}}{w}-x_{n}, \frac{t_{k_{n}+1}}{w}-x_{n}\right]^{2}\right)^{\nu / 2} \\
\leq & \left(\max \left\{\left(\frac{t_{k_{1}}}{w}-x_{1}\right)^{2},\left(\frac{t_{k_{1}+1}}{w}-x_{1}\right)^{2}\right\}\right. \\
& \left.+\cdots+\max \left\{\left(\frac{t_{k_{n}}}{w}-x_{n}\right)^{2},\left(\frac{t_{k_{n}+1}}{w}-x_{n}\right)^{2}\right\}\right)^{\nu / 2} \\
\leq & \frac{1}{w^{\nu}}\left(\max \left\{\left(w x_{1}-t_{k_{1}}\right)^{2},\left(w x_{1}-t_{k_{1}+1}\right)^{2}\right\}+\cdots\right. \\
& \left.+\max \left\{\left(w x_{n}-t_{k_{n}}\right)^{2},\left(w x_{n}-t_{k_{n}+1}\right)^{2}\right\}\right)^{\nu / 2} \\
\leq & \frac{1}{w^{\nu}}\left(\max \left\{\left\|w \underline{x}-t_{\underline{k}}\right\|_{2}^{2},\left\|w \underline{x}-t_{\underline{k}}-\Delta_{\underline{k}}\right\|_{2}^{2}\right\}+\cdots\right. \\
& \left.+\max \left\{\left\|w \underline{x}-t_{\underline{k}}\right\|_{2}^{2},\left\|w \underline{x}-t_{\underline{k}}-\Delta_{\underline{k}}\right\|_{2}^{2}\right\}\right)^{\nu / 2}
\end{aligned}
$$

where $\Delta_{\underline{k}}:=\left(\Delta_{k_{1}}, \ldots, \Delta_{k_{n}}\right)$. Now, recalling that each $\Delta_{k_{i}} \leq \Delta$, $i=1, \ldots, n$, we can observe that

$$
\left\|w \underline{x}-t_{\underline{k}}-\Delta_{\underline{k}}\right\|_{2} \leq\left\|w \underline{x}-t_{\underline{k}}\right\|_{2}+\sqrt{n} \Delta,
$$

then we finally obtain

$$
\max _{\underline{t} \in\left(R_{\underline{k}}^{w}-\underline{x}\right)}\|\underline{t}\|_{2}^{\nu} \leq w^{-\nu} n^{\nu / 2}\left[\left\|w \underline{x}-t_{\underline{k}}\right\|_{2}+\sqrt{n} \Delta\right]^{\nu} .
$$

Now, since $0<\nu \leq \beta \leq 1$, we have that the function $x^{\nu}$ for $x \geq 0$ is concave, and then subadditive, so we can write

$$
\max _{\underline{t} \in\left(R_{\underline{k}}^{w}-\underline{x}\right)}\|\underline{t}\|_{2}^{\nu} \leq w^{-\nu} n^{\nu / 2}\left[\left\|w \underline{x}-t_{\underline{k}}\right\|_{2}^{\nu}+n^{\nu / 2} \Delta^{\nu}\right] .
$$


Then, by the inequalities in (3) and (4), we obtain that

$$
\begin{aligned}
I_{1} & \leq w^{-\nu} n^{\nu / 2} C \sum_{\left\|w \underline{x}-t_{\underline{k}}\right\|_{2} \leq w \gamma / 2}\left|\chi\left(w \underline{x}-t_{\underline{k}}\right)\right|\left[\left\|w \underline{x}-t_{\underline{k}}\right\|_{2}^{\nu}+n^{\nu / 2} \Delta^{\nu}\right] \\
& \leq w^{-\nu} n^{\nu / 2} C\left[\sum_{\left\|w \underline{x}-t_{\underline{k}}\right\|_{2} \leq w \gamma / 2} \mid \chi\left(w \underline{x}-t_{\underline{k}}\right)\left\|w \underline{x}-t_{\underline{k}}\right\|_{2}^{\nu}+n^{\nu / 2} \Delta^{\nu} m_{0, \Pi^{n}}(\chi)\right] \\
& \leq w^{-\nu} n^{\nu / 2} C\left[m_{\nu, \Pi^{n}}(\chi)+n^{\nu / 2} \Delta^{\nu} m_{0, \Pi^{n}}(\chi)\right] .
\end{aligned}
$$

Now, by condition $(\chi 3)$ we have $m_{\beta, \Pi^{n}}(\chi)<+\infty$, and this implies that $m_{\nu, \Pi^{n}}(\chi)<+\infty$, for every $0<\nu \leq \beta$; moreover, by Lemma 3.1 (i), it turns out that $m_{0, \Pi^{n}}(\chi)<+\infty$. Hence, we can state

$$
I_{1}=\mathcal{O}\left(w^{-\nu}\right), \quad \text { as } w \rightarrow+\infty .
$$

Further, by Lemma 3.1 (ii),

$$
\begin{gathered}
I_{2} \leq 2\|f\|_{\infty} \sum_{\left\|w \underline{x}-t_{\underline{k}}\right\|_{2}>w \gamma / 2}\left|\chi\left(w \underline{x}-t_{\underline{k}}\right)\right|=\mathcal{O}\left(w^{-\beta}\right), \\
\text { as } w \rightarrow+\infty,
\end{gathered}
$$

uniformly with respect to $\underline{x} \in \mathbb{R}^{n}$, and finally from $(\chi 2)$ we obtain that $I_{3}=\mathcal{O}\left(w^{-\mu}\right)$, as $w \rightarrow+\infty$, uniformly with respect to $\underline{x} \in \mathbb{R}^{n}$. Thus, we have shown that

$$
\begin{aligned}
\left|\left(S_{w} f\right)(\underline{x})-f(\underline{x})\right| & \leq I_{1}+I_{2}+I_{3} \\
& =\mathcal{O}\left(w^{-\nu}\right)+\mathcal{O}\left(w^{-\beta}\right)+\mathcal{O}\left(w^{-\mu}\right), \quad \text { as } w \rightarrow+\infty,
\end{aligned}
$$

uniformly with respect to $\underline{x} \in \mathbb{R}^{n}$, and therefore we finally obtain that

$$
\left\|S_{w} f-f\right\|_{\infty}=\mathcal{O}\left(w^{-\epsilon}\right), \quad \text { as } w \rightarrow+\infty,
$$

where $\epsilon:=\min \{\nu, \beta, \mu\}$.

Now let $f \in \operatorname{Lip}_{\infty}(\nu)$, with $\beta \leq \nu \leq 1$ be fixed. Since $\operatorname{Lip}_{\infty}(\nu) \subseteq$ $\operatorname{Lip}_{\infty}(\beta)$, then for the previous case we can claim that

$$
\left\|S_{w} f-f\right\|_{\infty}=\mathcal{O}\left(w^{-\epsilon}\right), \quad \text { as } w \rightarrow+\infty,
$$

where $\epsilon:=\min \{\beta, \mu\}=\min \{\nu, \beta, \mu\}$, since $\nu \geq \beta$.

Finally, we consider $\chi$ satisfying condition $(\chi 3)$ for $\beta>1$. By the above considerations, $m_{\beta, \Pi}(\chi)<+\infty$ implies $m_{1, \Pi}(\chi)<+\infty$, which 
means that $\chi$ satisfies condition $(\chi 3)$ also for $\beta=1$, and so this case can be reduced to the previous step, and the assertion follows.

5. Order of approximation in Orlicz spaces $L^{\varphi}\left(\mathbb{R}^{n}\right)$. In order to study the behavior of the sampling Kantorovich operators when no necessary continuous signals (such as images) should be reconstructed, and to study their degree of approximation, we consider the case of the multivariate signal belonging to the general setting of Orlicz spaces $L^{\varphi}\left(\mathbb{R}^{n}\right)$, where $\varphi$ is a convex $\varphi$-function. We first recall the following modular continuity property for $S_{w}$.

Theorem 5.1. Let $\chi$ be a kernel. For every $f \in L^{\varphi}\left(\mathbb{R}^{n}\right)$, the following holds:

$$
I^{\varphi}\left[\lambda S_{w} f\right] \leq \frac{\|\chi\|_{1}}{\delta^{n} m_{0, \Pi^{n}}(\chi)} I^{\varphi}\left[\lambda m_{0, \Pi^{n}}(\chi) f\right] \quad(\lambda>0),
$$

for every $w>0$. In particular, $S_{w} f \in L^{\varphi}\left(\mathbb{R}^{n}\right)$ whenever $f \in L^{\varphi}\left(\mathbb{R}^{n}\right)$.

For a proof of Theorem 5.1, see [27]. The above theorem shows that the map $S_{w}: L^{\varphi}\left(\mathbb{R}^{n}\right) \rightarrow L^{\varphi}\left(\mathbb{R}^{n}\right)$ is well defined and continuous with respect to the modular topology $([\mathbf{3 9}])$.

Now, we establish the following result which gives a rate of approximation for the sampling Kantorovich operators in Orlicz spaces.

Theorem 5.2. Let $\chi$ be a kernel and $f \in L^{\varphi}\left(\mathbb{R}^{n}\right) \cap \operatorname{Lip} p_{\varphi}(\nu), 0<\nu \leq 1$. Suppose, in addition, that there exist $\theta, \gamma>0$ such that

$$
\int_{\|\underline{t}\|_{2} \leq \gamma} w^{n}|\chi(w \underline{t})|\|\underline{t}\|_{2}^{\nu} d \underline{t}=\mathcal{O}\left(w^{-\theta}\right), \quad \text { as } \quad w \rightarrow+\infty .
$$

Then there exists $\lambda>0$ such that

$$
I^{\varphi}\left[\lambda\left(S_{w} f-f\right)\right]=\mathcal{O}\left(w^{-\epsilon}\right), \quad \text { as } \quad w \rightarrow+\infty,
$$

with $\epsilon:=\min \{\theta, \nu, \mu, \alpha\}$, where $\mu, \alpha>0$ are the constants of conditions $(\chi 2)$ and $(\chi 4)$, respectively.

Proof. By the assumption $f \in L^{\varphi}\left(\mathbb{R}^{n}\right) \cap \operatorname{Lip}_{\varphi}(\nu), 0<\nu \leq 1$, we have that $I^{\varphi}\left[\lambda_{1} f\right]<+\infty$, and

$$
I^{\varphi}\left[\lambda_{2}(f(\cdot)-f(\cdot+\underline{t}))\right]=\mathcal{O}\left(\|\underline{t}\|_{2}^{\nu}\right), \quad \text { as } \quad\|\underline{t}\|_{2} \rightarrow 0,
$$


for some $\lambda_{1}, \lambda_{2}>0$. More in detail, there exist $M_{1}, \bar{\gamma}>0$ such that

$$
I^{\varphi}\left[\lambda_{2}(f(\cdot)-f(\cdot+\underline{t}))\right] \leq M_{1}\|\underline{t}\|_{2}^{\nu},
$$

for every $\|\underline{t}\|_{2} \leq \bar{\gamma}$. Now, by the properties of the convex modular functional $I^{\varphi}$, for $\lambda>0$ we can split the term $I^{\varphi}\left[\lambda\left(S_{w} f-f\right)\right]$ as follows:

$$
\begin{aligned}
I^{\varphi}\left[\lambda\left(S_{w} f-f\right)\right]= & \int_{\mathbb{R}^{n}} \varphi\left(\lambda\left|\left(S_{w} f\right)(\underline{x})-f(\underline{x})\right|\right) d \underline{x} \\
\leq & \frac{1}{3}\left\{\int _ { \mathbb { R } ^ { n } } \varphi \left(3 \lambda \mid\left(S_{w} f\right)(\underline{x})\right.\right. \\
& \left.-\sum_{\underline{k} \in \mathbb{Z}^{n}} \chi\left(w \underline{x}-t_{\underline{k}}\right) \frac{w^{n}}{A_{\underline{k}}} \int_{R_{\underline{k}}^{w}} f\left(\underline{u}+\underline{x}-\frac{t_{\underline{k}}}{w}\right) d \underline{u} \mid\right) d \underline{x} \\
& +\int_{\mathbb{R}^{n}} \varphi\left(3 \lambda \mid \sum_{\underline{k} \in \mathbb{Z}^{n}} \chi\left(w \underline{x}-t_{\underline{k}}\right)\right. \\
& \left.\times \frac{w^{n}}{A_{\underline{k}}} \int_{R_{\underline{k}}^{w}} f\left(\underline{u}+\underline{x}-\frac{t_{\underline{k}}}{w}\right) d \underline{u}-f(\underline{x}) A_{w}(\underline{x}) \mid\right) d \underline{x} \\
& \left.+\int_{\mathbb{R}^{n}} \varphi\left(3 \lambda\left|f(\underline{x}) A_{w}(\underline{x})-f(\underline{x})\right|\right) d \underline{x}\right\} \\
= & \frac{1}{3}\left\{J_{1}+J_{2}+J_{3}\right\} .
\end{aligned}
$$

In order to estimate the above terms, we begin considering the first one, namely $J_{1}$. Applying Jensen's inequality (see, e.g., [26]) and the Fubini-Tonelli theorem,

$$
\begin{aligned}
J_{1}= & \int_{\mathbb{R}^{n}} \varphi\left(3 \lambda \mid\left(S_{w} f\right)(\underline{x})-\sum_{\underline{k} \in \mathbb{Z}^{n}} \chi\left(w \underline{x}-t_{\underline{k}}\right)\right. \\
& \left.\times \frac{w^{n}}{A_{\underline{k}}} \int_{R_{\underline{k}}^{w}} f\left(\underline{u}+\underline{x}-\frac{t_{\underline{k}}}{w}\right) d \underline{u} \mid\right) d \underline{x} \\
\leq & \frac{1}{m_{0, \Pi^{n}}(\chi)} \int_{\mathbb{R}^{n}} \sum_{\underline{k} \in \mathbb{Z}^{n}}\left|\chi\left(w \underline{x}-t_{\underline{k}}\right)\right| \\
& \times \varphi\left(3 \lambda m_{0, \Pi^{n}}(\chi) \frac{w^{n}}{A_{\underline{k}}} \int_{R_{\underline{k}}^{w}}\left|f(\underline{u})-f\left(\underline{u}+\underline{x}-\frac{t_{k}}{w}\right)\right| d \underline{u}\right) d \underline{x} \\
\leq & \frac{1}{m_{0, \Pi^{n}}(\chi)} \sum_{\underline{k} \in \mathbb{Z}^{n}} \int_{\mathbb{R}^{n}}\left|\chi\left(w \underline{x}-t_{\underline{k}}\right)\right|
\end{aligned}
$$




$$
\times \varphi\left(3 \lambda m_{0, \Pi^{n}}(\chi) \frac{w^{n}}{A_{\underline{k}}} \int_{R_{\underline{k}}^{w}}\left|f(\underline{u})-f\left(\underline{u}+\underline{x}-\frac{t_{\underline{k}}}{w}\right)\right| d \underline{u}\right) d \underline{x} .
$$

By the change of variable $\underline{t}=\underline{x}-t_{k} / w$, applying the Fubini-Tonelli theorem and Jensen's inequality again, we may obtain the following:

$$
\begin{aligned}
& J_{1} \leq \frac{1}{m_{0, \Pi^{n}}(\chi)} \int_{\mathbb{R}^{n}}|\chi(w \underline{t})| \\
& \sum_{\underline{k} \in \mathbb{Z}^{n}} \varphi\left(3 \lambda m_{0, \Pi^{n}}(\chi) \frac{w^{n}}{A_{\underline{k}}} \int_{R_{\underline{k}}^{w}}|f(\underline{u})-f(\underline{u}+\underline{t})| d \underline{u}\right) d \underline{t} \\
& \leq \frac{1}{m_{0, \Pi^{n}}(\chi)} \int_{\mathbb{R}^{n}}|\chi(w \underline{t})| \\
& \times\left\{\sum_{\underline{k} \in \mathbb{Z}^{n}} \frac{w^{n}}{A_{\underline{k}}} \int_{R_{\underline{k}}^{w}} \varphi\left(3 \lambda m_{0, \Pi^{n}}(\chi)|f(\underline{u})-f(\underline{u}+\underline{t})|\right) d \underline{u}\right\} d \underline{t} \\
& \leq \frac{1}{m_{0, \Pi^{n}}(\chi) \delta^{n}} \int_{\mathbb{R}^{n}} w^{n} \\
& \times|\chi(w \underline{t})|\left\{\sum_{k \in \mathbb{Z}} \int_{R_{\underline{k}}^{w}} \varphi\left(3 \lambda m_{0, \Pi^{n}}(\chi)|f(\underline{u})-f(\underline{u}+\underline{t})|\right) d \underline{u}\right\} d \underline{t} \\
& =\frac{1}{m_{0, \Pi^{n}}(\chi) \delta^{n}} \int_{\mathbb{R}^{n}} w^{n} \\
& \times|\chi(w \underline{t})|\left\{\int_{\mathbb{R}^{n}} \varphi\left(3 \lambda m_{0, \Pi^{n}}(\chi)|f(\underline{u})-f(\underline{u}+\underline{t})|\right) d \underline{u}\right\} d \underline{t} \\
& =\frac{1}{m_{0, \Pi^{n}}(\chi) \delta^{n}}\left\{\int_{\|\underline{t}\|_{2} \mid \leq \widetilde{\gamma}} w^{n}\right. \\
& \times|\chi(w \underline{t})|\left(\int_{\mathbb{R}^{n}} \varphi\left(3 \lambda m_{0, \Pi^{n}}(\chi)|f(\underline{u})-f(\underline{u}+\underline{t})|\right) d \underline{u}\right) d \underline{t}+\int_{\|t\|_{2}>\widetilde{\gamma}} w^{n} \\
& \left.\times|\chi(w \underline{t})|\left(\int_{\mathbb{R}^{n}} \varphi\left(3 \lambda m_{0, \Pi^{n}}(\chi)|f(\underline{u})-f(\underline{u}+\underline{t})|\right) d \underline{u}\right) d \underline{t}\right\} \\
& =\frac{1}{m_{0, \Pi^{n}}(\chi) \delta^{n}}\left\{J_{1,1}+J_{1,2}\right\},
\end{aligned}
$$

with $\widetilde{\gamma}:=\min \{\gamma, \bar{\gamma}\}$, where $\gamma>0$ is the constant condition (5). Now, without any loss of generality, we can choose $\lambda>0$ sufficiently small, 
such that:

$$
\lambda \leq \min \left\{\frac{\lambda_{1}}{3 M_{2}}, \frac{\lambda_{1}}{6 m_{0, \Pi^{n}}(\chi)}, \frac{\lambda_{2}}{3 m_{0, \Pi^{n}}(\chi)}, \lambda_{2} \delta^{n} / 3 \Delta^{n} m_{0, \Pi^{n}}(\chi)\right\},
$$

where $M_{2}>0$ is a suitable positive constant obtained from condition $(\chi 2)$, i.e.,

$$
\left|\sum_{k \in \mathbb{Z}} \chi\left(w \underline{x}-t_{\underline{k}}\right)-1\right| \leq M_{2} w^{-\mu}
$$

uniformly with respect to $\underline{x} \in \mathbb{R}^{n}$ and for sufficiently large $w>0$. Now, recalling that $f \in \operatorname{Lip}_{\varphi}(\nu)$, by condition (5), it is easy to deduce the following estimate:

$$
\begin{aligned}
J_{1,1} & \leq \int_{\|\underline{t}\|_{2} \leq \widetilde{\gamma}} w^{n}|\chi(w \underline{t})|\left[\int_{\mathbb{R}^{n}} \varphi\left(\lambda_{2}|f(\underline{u})-f(\underline{u}+\underline{t})|\right) d \underline{u}\right] d \underline{t} \\
& \leq M_{1} \int_{\|\underline{t}\|_{2} \leq \widetilde{\gamma}} w^{n}|\chi(w \underline{t})|\|\underline{t}\|_{2}^{\nu} d \underline{t} \\
& =\mathcal{O}\left(w^{-\theta}\right), \quad \text { as } \quad w \rightarrow+\infty,
\end{aligned}
$$

while, in the case of $J_{1,2}$, by the convexity of $\varphi$, we have

$$
\begin{aligned}
J_{1,2} \leq & \int_{\|\underline{t}\|_{2}>\widetilde{\gamma}} w^{n}|\chi(w \underline{t})| \frac{1}{2}\left[\int_{\mathbb{R}^{n}} \varphi\left(6 \lambda m_{0, \Pi^{n}}(\chi)|f(\underline{u})|\right) d \underline{u}\right. \\
& \left.+\int_{\mathbb{R}^{n}} \varphi\left(6 \lambda m_{0, \Pi^{n}}(\chi)|f(\underline{u}+\underline{t})|\right) d \underline{u}\right] d \underline{t} .
\end{aligned}
$$

Observing that

$$
\int_{\mathbb{R}^{n}} \varphi\left(6 \lambda m_{0, \Pi^{n}}(\chi)|f(\underline{u}+\underline{t})|\right) d \underline{u}=\int_{\mathbb{R}^{n}} \varphi\left(6 \lambda m_{0, \Pi^{n}}(\chi)|f(\underline{u})|\right) d \underline{u},
$$

for every $\underline{t} \in \mathbb{R}^{n}$, it turns out

$$
\begin{aligned}
J_{1,2} & \leq \int_{\|\underline{t}\|_{2}>\widetilde{\gamma}} w^{n}|\chi(w \underline{t})| \int_{\mathbb{R}^{n}} \varphi\left(6 \lambda m_{0, \Pi^{n}}(\chi)|f(\underline{u})|\right) d \underline{u} \\
& =I^{\varphi}\left[6 \lambda m_{0, \Pi^{n}}(\chi) f\right] \int_{\|\underline{t}\|_{2}>\widetilde{\gamma}} w^{n}|\chi(w \underline{t})| d \underline{t} .
\end{aligned}
$$

Therefore, by the above inequality, assumptions and considerations, we deduce that

$$
J_{1,2}=\mathcal{O}\left(w^{-\alpha}\right), \quad \text { as } w \rightarrow+\infty
$$


Now, we estimate $J_{2}$. Setting $\underline{t}=\underline{u}-t_{\underline{k}} / w$, we have

$$
\begin{aligned}
J_{2} & \leq \int_{\mathbb{R}^{n}} \varphi\left(3 \lambda \mid \sum_{\underline{k} \in \mathbb{Z}^{n}} \chi\left(w \underline{x}-t_{\underline{k}}\right)\left[\frac{w^{n}}{A_{\underline{k}}} \int_{R_{\underline{w}}^{w}} f\left(\underline{u}+\underline{x}-\frac{t_{\underline{k}}}{w}\right) d \underline{u}-f(\underline{x})\right]\right) d \underline{x} \\
& =\int_{\mathbb{R}^{n}} \varphi\left(3 \lambda \mid \sum_{\underline{k} \in \mathbb{Z}^{n}} \chi\left(w \underline{x}-t_{\underline{k}}\right)\left[\frac{w^{n}}{A_{\underline{k}}} \int_{\left(R_{\underline{k}}^{w}-t_{\underline{k}} / w\right)} f(\underline{x}+\underline{t}) d \underline{t}-f(\underline{x})\right]\right) d \underline{x},
\end{aligned}
$$

where $\left(R_{k}^{w}-t_{\underline{k}} / w\right):=\left[0, \Delta_{k_{1}} / w\right] \times \cdots \times\left[0, \Delta_{k_{n}} / w\right]$, for every $\underline{k} \in \mathbb{Z}^{n}$ and $w>\overline{0}$. Thus,

$$
\begin{aligned}
J_{2} & \left.\leq \int_{\mathbb{R}^{n}} \varphi\left(3 \lambda \mid \sum_{\underline{k} \in \mathbb{Z}^{n}} \chi\left(w \underline{x}-t_{\underline{k}}\right) \frac{w^{n}}{A_{\underline{k}}} \int_{\left(R_{\underline{k}}^{w}-t_{\underline{k}} / w\right)}[f(\underline{x}+\underline{t})-f(\underline{x})] d \underline{t}\right]\right) d \underline{x} \\
& \leq \int_{\mathbb{R}^{n}} \varphi\left(3 \lambda\left[\sum_{\underline{k} \in \mathbb{Z}^{n}}\left|\chi\left(w \underline{x}-t_{\underline{k}}\right)\right| \frac{w^{n}}{\delta^{n}} \int_{\left(R_{\underline{\underline{w}}}^{w}-t_{\underline{k}} / w\right)}|f(\underline{x}+\underline{t})-f(\underline{x})| d \underline{t}\right]\right) d \underline{x} \\
& \leq \int_{\mathbb{R}^{n}} \varphi\left(3 \lambda\left[\sum_{\underline{k} \in \mathbb{Z}^{n}}\left|\chi\left(w \underline{x}-t_{\underline{k}}\right)\right| \frac{w^{n}}{\delta^{n}} \int_{\left(\Delta_{w}\right)}|f(\underline{x}+\underline{t})-f(\underline{x})| d \underline{t}\right]\right) d \underline{x},
\end{aligned}
$$

where $\left(\Delta_{w}\right):=[0, \Delta / w] \times \cdots \times[0, \Delta / w]$. Then, using Jensen's inequality, the Fubini-Tonelli theorem, and since $f \in \operatorname{Lip}_{\varphi}(\nu), 0<$ $\nu \leq 1$, for sufficiently large $w>0$, we can write

$$
\begin{aligned}
J_{2} & \leq \int_{\mathbb{R}^{n}} \varphi\left(3 \lambda m_{0, \Pi^{n}}(\chi) \frac{w^{n}}{\delta^{n}} \int_{\left(\Delta_{w}\right)}|f(\underline{x}+\underline{t})-f(\underline{x})| d \underline{t}\right) d \underline{x}, \\
& \leq \int_{\mathbb{R}^{n}} \frac{w^{n}}{\Delta^{n}}\left[\int_{\left(\Delta_{w}\right)} \varphi\left(3 \lambda m_{0, \Pi^{n}}(\chi) \frac{\Delta^{n}}{\delta^{n}}|f(\underline{x}+\underline{t})-f(\underline{x})|\right) d \underline{t}\right] d \underline{x}, \\
& =\frac{w^{n}}{\Delta^{n}} \int_{\left(\Delta_{w}\right)}\left[\int_{\mathbb{R}^{n}} \varphi\left(3 \lambda m_{0, \Pi^{n}}(\chi) \frac{\Delta^{n}}{\delta^{n}}|f(\underline{x}+\underline{t})-f(\underline{x})|\right) d \underline{x}\right] d \underline{t} \\
& \leq \frac{w^{n}}{\Delta^{n}} \int_{\left(\Delta_{w}\right)}\left[\int_{\mathbb{R}^{n}} \varphi\left(\lambda_{2}|f(\underline{x}+\underline{t})-f(\underline{x})|\right) d \underline{x}\right] d \underline{t} \\
& \leq M_{1} \frac{w^{n}}{\Delta^{n}} \int_{\left(\Delta_{w}\right)}\|\underline{t}\|_{2}^{\nu} d \underline{t},
\end{aligned}
$$

being $\|\underline{t}\|_{2} \leq \bar{\gamma}$. By the change of variable $\underline{t}=\underline{u} / w$, and denoting by 
$(\bar{\Delta}):=[0, \Delta] \times \cdots \times[0, \Delta]$, we have that

$$
J_{2} \leq \frac{M_{1}}{\Delta^{n}} \int_{(\bar{\Delta})}\|\underline{u} / w\|_{2}^{\nu} d \underline{u}=w^{-\nu} \frac{M_{1}}{\Delta^{n}} \int_{(\bar{\Delta})}\|\underline{u}\|_{2}^{\nu} d \underline{u}=: C w^{-\nu},
$$

for sufficiently large $w>0$, i.e., $J_{2}=\mathcal{O}\left(w^{-\nu}\right)$, as $w \rightarrow+\infty$.

Finally, we proceed by estimating the last term of the initial inequality, namely, $J_{3}$. Recalling condition $(\chi 2)$ and the convexity of $\varphi$, for sufficiently large $w>0$, it is easy to obtain

$$
\begin{aligned}
J_{3} & \leq \int_{\mathbb{R}^{n}} \varphi\left(3 \lambda|f(\underline{x})|\left|A_{w}(\underline{x})-1\right| \mid\right) d \underline{x} \\
& \leq \int_{\mathbb{R}^{n}} \varphi\left(3 \lambda M_{2} w^{-\mu} \mid f(\underline{x}) \|\right) d \underline{x} \\
& \leq w^{-\mu} I^{\varphi}\left[3 M_{2} \lambda f\right] \leq w^{-\mu} I^{\varphi}\left[\lambda_{1} f\right]<+\infty .
\end{aligned}
$$

Thus, combining all the above estimates, we can conclude that

$$
I^{\varphi}\left[\lambda\left(S_{w} f-f\right)\right]=\mathcal{O}\left(w^{-\epsilon}\right), \quad \text { as } w \rightarrow+\infty,
$$

where $\epsilon:=\min \{\theta, \nu, \mu, \alpha\}$.

The assumptions required in the above theorem are rather standard when the problem of the rate of approximation for a family of linear discrete operators is studied in Orlicz spaces. In particular, condition (5) represents a relation between the kernel of $S_{w}$ and the Lipschitz class under consideration. Condition (5) is clearly satisfied when, for instance, the kernel $\chi$ is with compact support. Denoting by $B(\underline{0}, R)$ the closed ball centered in the origin and with radius $R>0$, if supp $\chi \subset B(\underline{0}, R)$, we have

(6) $\int_{\|\underline{t}\|_{2} \leq \gamma} w^{n}|\chi(w \underline{t})|\|t\|_{2}^{\nu} d \underline{t} \leq \int_{\|\underline{u}\|_{2} \leq R}|\chi(\underline{u})|\|\underline{u} / w\|_{2}^{\nu} d \underline{u}=: K w^{-\nu}$,

for sufficiently large $w>0$, i.e., $\theta=\nu$. Moreover, in the above case, $\chi$ satisfies condition $(\chi 4)$ for every $\alpha>0$. We obtain the following

Corollary 5.3. Let $\chi$ be a kernel with compact support. Moreover, let $f \in L^{\varphi}\left(\mathbb{R}^{n}\right) \cap \operatorname{Lip}_{\varphi}(\nu), 0<\nu \leq 1$. Then, there exists a $\lambda>0$ such that

$$
I^{\varphi}\left[\lambda\left(S_{w} f-f\right)\right]=\mathcal{O}\left(w^{-\epsilon}\right), \quad \text { as } w \rightarrow+\infty,
$$

with $\epsilon:=\min \{\nu, \mu\}$, where $\mu>0$ is the constant of condition $(\chi 2)$. 
Condition (5) is satisfied also in the case of kernels with unbounded support if we require an additional condition on $\chi$, i.e., that the multivariate absolute moment

$$
m_{\nu}(\chi):=\int_{\mathbb{R}^{n}}|\chi(\underline{u})|\|\underline{u}\|_{2}^{\nu} d \underline{u}<+\infty,
$$

for some $0<\nu \leq 1$. Indeed, if condition (7) holds, for every $\gamma>0$ we have:

$$
\int_{\|\underline{t}\|_{2} \leq \gamma} w^{n}|\chi(w \underline{t})|\|\underline{t}\|_{2}^{\nu} d \underline{t} \leq \int_{\|\underline{u}\|_{2} \leq \gamma w}|\chi(\underline{u})|\|\underline{u} / w\|_{2}^{\nu} d \underline{u} \leq m_{\nu}(\chi) w^{-\nu},
$$

for every $w>0$, which shows that (5) holds for $\theta=\nu$. Thus, we obtain the following

Corollary 5.4. Let $\chi$ be a kernel with $m_{\nu}(\chi)<+\infty$, for some $0<\nu \leq 1$. Moreover, let $f \in L^{\varphi}\left(\mathbb{R}^{n}\right) \cap \operatorname{Lip}_{\varphi}(\nu)$ be fixed. Then there exists $\lambda>0$ such that

$$
I^{\varphi}\left[\lambda\left(S_{w} f-f\right)\right]=\mathcal{O}\left(w^{-\epsilon}\right), \quad \text { as } w \rightarrow+\infty,
$$

with $\epsilon:=\min \{\nu, \mu, \alpha\}$, where $\mu>0$ and $\alpha>0$ are the constants of conditions $(\chi 2)$ and $(\chi 4)$, respectively.

Remark 5.5. Examples of convex $\varphi$-functions generating Orlicz spaces, where the theory of multivariate sampling Kantorovich operators holds, are: $\varphi_{p}(u):=u^{p}, 1 \leq p<+\infty$, which generates the well-known $L^{p}\left(\mathbb{R}^{n}\right)$ spaces, $\varphi_{\alpha, \beta}:=u^{\alpha} \log ^{\beta}(u+e)$, for $\alpha \geq 1, \beta>0$, which gives rise to interpolation spaces and finally, $\varphi_{\gamma}(u)=e^{u^{\gamma}}-1$, for $\gamma>0, u \geq 0$, in order to obtain the exponential spaces. It is well known that the modular functional corresponding to $\varphi_{p}(u)$ is $I^{\varphi_{p}}[f]:=\|f\|_{p}^{p}$. The modular functionals corresponding to $\varphi_{\alpha, \beta}$ and $\varphi_{\gamma}$ are

$$
I^{\varphi_{\alpha, \beta}}[f]:=\int_{\mathbb{R}^{n}}|f(\underline{x})|^{\alpha} \log ^{\beta}(e+|f(\underline{x})|) d \underline{x}, \quad\left(f \in M\left(\mathbb{R}^{n}\right)\right),
$$

and

$$
I^{\varphi_{\gamma}}[f]:=\int_{\mathbb{R}^{n}}\left(e^{|f(\underline{x})|^{\gamma}}-1\right) d \underline{x}, \quad\left(f \in M\left(\mathbb{R}^{n}\right)\right),
$$

respectively. The $L^{\alpha} \log ^{\beta} L$-spaces (interpolation or Zygmund spaces generated by $\left.\varphi_{\alpha, \beta}\right)$, are widely used in the theory of partial differential 
equations, while the exponential spaces (generated by $\varphi_{\gamma}$ ) are important for embedding theorems between Sobolev spaces.

6. Applications to special kernels. One important fact in our theory is the choice of the kernels, which influence the order of approximation that can be achieved by our operators (see e.g., $[\mathbf{2 9}, \mathbf{3 0}]$ in one-dimensional setting).

To construct, in general, kernels satisfying all the assumptions $\left(\chi_{i}\right)$, $i=1, \ldots, 4$, is not very easy.

For this reason, here we show a procedure useful to construct examples using product of univariate kernels, see, e.g., [12, 17, 27, 28]. For the sake of simplicity, we consider only the case of uniform sampling scheme, i.e., $t_{\underline{k}}=\underline{k}$.

Denote by $\chi_{1}, \ldots, \chi_{n}$, the univariate functions $\chi_{i}: \mathbb{R} \rightarrow \mathbb{R}, \chi_{i} \in$ $L^{1}(\mathbb{R})$ and are bounded in a neighborhood of $0 \in \mathbb{R}$, satisfying the following assumptions:

$$
m_{\beta, \Pi^{1}}\left(\chi_{i}\right):=\sup _{x \in \mathbb{R}} \sum_{k \in \mathbb{Z}}\left|\chi_{i}(x-k)\right| \cdot|x-k|^{\beta}<+\infty,
$$

$i=1, \ldots, n$, for some $\beta>0 ;$ moreover,

$$
\sum_{k \in \mathbb{Z}} \chi_{i}(x-k)=1
$$

for every $x \in \mathbb{R}, i=1, \ldots, n$, and for every $\widetilde{M}>0$

$$
\int_{|x|>\widetilde{M}} w\left|\chi_{i}(w x)\right| d x=\mathcal{O}\left(w^{-\alpha}\right), \quad \text { as } w \rightarrow+\infty,
$$

for every $i=1, \ldots, n$ and for some $\alpha>0$.

Remark 6.1. Note that condition (10) is equivalent to

$$
\widehat{\chi}(k):= \begin{cases}0, & k \in \mathbb{Z} \backslash\{0\}, \\ 1, & k=0\end{cases}
$$

where $\widehat{\chi}(v):=\int_{\mathbb{R}} \chi(u) e^{-i v u} d u, v \in \mathbb{R}$, denotes the Fourier transform of $\chi$; see $[3,13,27,29]$.

Now, setting 


$$
\chi(\underline{x}):=\prod_{i=1}^{n} \chi_{i}\left(x_{i}\right), \quad \underline{x}=\left(x_{1}, \ldots, x_{n}\right) \in \mathbb{R}^{n},
$$

we can prove that $\chi$ is a multivariate kernel for the operators $S_{w}$ satisfying all the assumptions of our theory. Indeed, we have that $\chi \in L^{1}\left(\mathbb{R}^{n}\right)$ since

$$
\begin{aligned}
\int_{\mathbb{R}^{n}}|\chi(\underline{x})| d \underline{x} & =\int_{\mathbb{R}^{n}} \prod_{i=1}^{n}\left|\chi_{i}\left(x_{i}\right)\right| d x_{1} \ldots d x_{n} \\
& =\prod_{i=1}^{n} \int_{\mathbb{R}}\left|\chi_{i}\left(x_{i}\right)\right| d x_{i}=\prod_{i=1}^{n}\left\|\chi_{i}\right\|_{1}<+\infty .
\end{aligned}
$$

Moreover, it is also obviously bounded in a neighborhood of the origin; then condition $(\chi 1)$ holds. Further, by condition (10),

$$
A_{w}(\underline{x})=\sum_{\underline{k} \in \mathbb{Z}^{n}} \chi(w \underline{x}-\underline{k})=\prod_{i=1}^{n} \sum_{k_{i} \in \mathbb{Z}} \chi_{i}\left(w x_{i}-k_{i}\right)=1,
$$

then $A_{w}(\underline{x})-1=0$, for every $\underline{x} \in \mathbb{R}^{n}$ and $w>0$, i.e., $\chi$ satisfies condition $(\chi 2)$ for every $\mu>0$. Concerning condition $(\chi 3)$, we have:

$$
\begin{aligned}
m_{\beta, \Pi^{n}}(\chi) & =\sup _{\underline{x} \in \mathbb{R}^{n}} \sum_{\underline{k} \in \mathbb{Z}^{n}}|\chi(\underline{x}-\underline{k})| \cdot\|\underline{x}-\underline{k}\|_{2}^{\beta} \\
& \leq K \sup _{\underline{x} \in \mathbb{R}^{n}} \sum_{\underline{k} \in \mathbb{Z}^{n}}|\chi(\underline{x}-\underline{k})| \cdot\|\underline{x}-\underline{k}\|^{\beta},
\end{aligned}
$$

where $\|\underline{x}\|:=\max \left\{\left|x_{i}\right|, \quad i=1, \ldots, n\right\}, \underline{x} \in \mathbb{R}^{n}$, and $K>0$ is a suitable constant (we recall that all the norms are equivalent in $\mathbb{R}^{n}$ ). Denoting by $\underline{k}_{[j]} \in \mathbb{Z}^{n-1}$ the vectors $\underline{k}_{[j]}:=\left(k_{1}, \ldots, k_{j-1}, k_{j+1}, \ldots, k_{n}\right)$ and by $\Pi_{[j]}^{n-1}$ the restriction of the sequence $\Pi^{n}$ to the vectors $\underline{k}_{[j]}$, with $\underline{k}_{[j]} \in \mathbb{Z}^{n-1}$, then we can write

$$
\begin{aligned}
m_{\beta, \Pi^{n}}(\chi) & \leq K \sup _{\underline{x} \in \mathbb{R}^{n}}\left[\sum_{\underline{k} \in \mathbb{Z}^{n}}|\chi(\underline{x}-\underline{k})| \cdot\left(\sum_{j=1}^{n}\left|x_{j}-k_{j}\right|^{\beta}\right)\right] \\
& \leq K \sup _{\underline{x} \in \mathbb{R}^{n}} \sum_{j=1}^{n}\left[\sum_{\underline{k} \in \mathbb{Z}^{n}}|\chi(\underline{x}-\underline{k})| \cdot\left|x_{j}-k_{j}\right|^{\beta}\right]
\end{aligned}
$$




$$
\begin{aligned}
& \leq K \sup _{\underline{x} \in \mathbb{R}^{n}} \sum_{j=1}^{n}\left[\sum_{\underline{k} \in \mathbb{Z}^{n}}\left(\prod_{i=1}^{n}\left|\chi_{i}\left(x_{i}-k_{i}\right)\right|\right) \cdot\left|x_{j}-k_{j}\right|^{\beta}\right] \\
& \leq \operatorname{Sup}_{\underline{x} \in \mathbb{R}^{n}} \sum_{j=1}^{n}\left[\sum_{\underline{k}_{[j]} \in \mathbb{Z}^{n-1}}\left(\prod_{\substack{i=1 \\
i \neq j}}^{n}\left|\chi_{i}\left(x_{i}-k_{i}\right)\right|\right) \sum_{k_{j} \in \mathbb{Z}}\left|\chi_{j}\left(x_{j}-k_{j}\right)\right|\left|x_{j}-k_{j}\right|^{\beta}\right] \\
& \leq K \sup _{\underline{x} \in \mathbb{R}^{n}} \sum_{j=1}^{n}\left[\sum_{\underline{k}_{[j]} \in \mathbb{Z}^{n-1}}\left(\prod_{\substack{i=1 \\
i \neq j}}^{n}\left|\chi_{i}\left(x_{i}-k_{i}\right)\right|\right) m_{\beta, \Pi^{1}}\left(\chi_{j}\right)\right] \\
& \leq K \sup _{\underline{x} \in \mathbb{R}^{n}} \sum_{j=1}^{n}\left[\prod_{\substack{i=1 \\
i \neq j}}^{n}\left(\sum_{\underline{k}_{[j]} \in \mathbb{Z}^{n-1}}\left|\chi_{i}\left(x_{i}-k_{i}\right)\right|\right) m_{\beta, \Pi^{1}}\left(\chi_{j}\right)\right] \\
& \leq K \sup _{\underline{x} \in \mathbb{R}^{n}} \sum_{j=1}^{n}\left[\prod_{\substack{i=1 \\
i \neq j}}^{n} m_{0, \Pi_{[j]}^{n-1}}\left(\chi_{i}\right) \cdot m_{\beta, \Pi^{1}}\left(\chi_{j}\right)\right] \\
& =K \sum_{j=1}^{n}\left[\prod_{\substack{i=1 \\
i \neq j}}^{n} m_{0, \Pi_{[j]}^{n-1}}\left(\chi_{i}\right) \cdot m_{\beta, \Pi^{1}}\left(\chi_{j}\right)\right]<+\infty .
\end{aligned}
$$

Finally, for every $M>0$, there exists a suitable constant $\widetilde{M}>0$ such that

$$
\begin{aligned}
& \int_{\|\underline{u}\|_{2}>M} w^{n}|\chi(w \underline{u})| d \underline{u} \leq \int_{\|\underline{u}\|>\widetilde{M}} w^{n}|\chi(w \underline{u})| d \underline{u}=\int_{\|\underline{u}\|>\widetilde{M}}\left[\prod_{i=1}^{n} w\left|\chi_{i}\left(w u_{i}\right)\right|\right] d \underline{u} \\
& \leq \sum_{j=1}^{n}\left\{\left[\int_{\left\|\underline{u}_{[j]}\right\|>\widetilde{M}} \prod_{\substack{i=1 \\
i \neq j}}^{n} w\left|\chi_{i}\left(w u_{i}\right)\right| d u_{[j]}\right]\right. \\
&\left.\cdot \int_{\left|u_{j}\right|>\widetilde{M}} w\left|\chi_{j}\left(w u_{j}\right)\right| d u_{j}\right\} \\
& \leq \sum_{j=1}^{n}\left\{\left[\int_{\left\|\underline{t}_{[j]}\right\|>w \widetilde{M}} \prod_{\substack{i=1 \\
i \neq j}}^{n}\left|\chi_{i}\left(t_{i}\right)\right| d \underline{t}_{[j]}\right]\right. \\
&\left.\cdot \int_{\left|u_{j}\right|>\widetilde{M}} w\left|\chi_{j}\left(w u_{j}\right)\right| d u_{j}\right\}
\end{aligned}
$$




$$
\begin{aligned}
& \leq \sum_{j=1}^{n}\left\{\left[\int_{\mathbb{R}^{n-1}} \prod_{\substack{i=1 \\
i \neq j}}^{n}\left|\chi_{i}\left(t_{i}\right)\right| d \underline{t}_{[j]}\right]\right] \\
& \left.\cdot \int_{\left|u_{j}\right|>\widetilde{M}} w\left|\chi_{j}\left(w u_{j}\right)\right| d u_{j}\right\} \\
& \leq \sum_{j=1}^{n}\left\{\left[\prod_{\substack{i=1 \\
i \neq j}} \int_{\mathbb{R}^{n-1}}\left|\chi_{i}\left(t_{i}\right)\right| d \underline{t}_{[j]}\right]\right. \\
& \leq \sum_{j=1}^{n}\left\{\left(\prod_{\substack{i=1 \\
i \neq j}}^{n} \cdot \int_{\left|u_{j}\right|>\widetilde{M}} w\left|\chi_{j}\left(w u_{j}\right)\right| d u_{j}\right\}\right. \\
& \left|u_{j}\right|>\widetilde{M} \\
&
\end{aligned}
$$

where we used the change of variable $w \underline{u}_{[j]}=\underline{t}_{[j]}$; then, in correspondence to the constant $\alpha>0$ of condition (11) the following holds:

$$
\int_{\|\underline{u}\|_{2}>M} w^{n}|\chi(w \underline{u})| d \underline{u}=\mathcal{O}\left(w^{-\alpha}\right), \quad \text { as } w \rightarrow+\infty,
$$

for every $M>0$, i.e., $\chi$ satisfies condition $(\chi 4)$ with $\alpha>0$. Thus, we can say that $\chi$ is a multivariate kernel.

Now, we will show some practical examples of multivariate kernels, constructed by product of univariate kernels.

In what follows, we denote by

$$
F(x):=\frac{1}{2} \operatorname{sinc}^{2}\left(\frac{x}{2}\right) \quad(x \in \mathbb{R}),
$$

the well known one-dimensional Fejér's kernel. The sinc $(x)$ function is defined by

$$
\operatorname{sinc}(x):=\left\{\begin{array}{cl}
\frac{\sin \pi x}{\pi x} & x \in \mathbb{R} \backslash\{0\} \\
1 & x=0 .
\end{array}\right.
$$

It is easy to observe that the function $F$ is bounded, belongs to $L^{1}(\mathbb{R})$ and satisfies the moment conditions (9) for every $0<\beta \leq 1$. For the above properties see, e.g., $[\mathbf{3}, \mathbf{1 3}, \mathbf{2 9}, \mathbf{3 0}]$. Moreover, it is also possible 
to observe that the Fourier transform of $F$ is given by (see [13])

$$
\widehat{F}(v):=\left\{\begin{array}{l}
1-|v / \pi|, \quad|v| \leq \pi, \\
0, \quad|v|>\pi,
\end{array}\right.
$$

and therefore condition (10) is fulfilled as a consequence of Remark 6.1. In addition,

$$
\int_{|u|>\widetilde{M}} w F(w u) d u \leq \frac{2}{\pi^{2}} w^{-1} \int_{|u|>\widetilde{M}} \frac{1}{u^{2}} d u=: K w^{-1},
$$

for every $\widetilde{M}>0$ and $w>0$, and hence condition (11) holds for $\alpha=1$. Finally, in [13], it is proved that Fejér's kernel satisfies the finiteness of the absolute moments, i.e., $m_{\nu}(F)<+\infty$, for every $0<\nu \leq 1$. Then, according to the procedure described in this section, we can define by

$$
\mathcal{F}_{n}(\underline{x})=\prod_{i=1}^{n} F\left(x_{i}\right), \quad \underline{x}=\left(x_{1}, \ldots, x_{n}\right) \in \mathbb{R}^{n},
$$

the multivariate Fejér's kernel, which satisfies the conditions upon a multivariate kernel with $m_{\nu}\left(\mathcal{F}_{n}\right)<+\infty$, for every $0<\nu \leq 1$. Then the multivariate sampling Kantorovich operators based on the Fejér's kernel, in the case of uniform sampling, now take the form

$$
\left(S_{w}^{\mathcal{F}_{n}} f\right)(\underline{x})=\sum_{\underline{k} \in \mathbb{Z}^{n}}\left[w^{n} \int_{R_{\underline{\underline{w}}}^{w}} f(\underline{u}) d \underline{u}\right] \mathcal{F}_{n}(w \underline{x}-\underline{k}), \quad\left(\underline{x} \in \mathbb{R}^{n}\right),
$$

for every $w>0$, where $f: \mathbb{R}^{n} \rightarrow \mathbb{R}$ is a locally integrable function such that the above series is convergent for every $\underline{x} \in \mathbb{R}^{n}$. For $S_{w}^{\mathcal{F}_{n}} f$, from Theorem 4.1, Theorem 5.1 and Corollary 5.4, we can obtain respectively the following

Corollary 6.2. Let $f \in \operatorname{Lip}_{\infty}(\nu)$, with $0<\nu \leq 1$. Then

$$
\left\|S_{w}^{\mathcal{F}_{n}} f-f\right\|_{\infty}=\mathcal{O}\left(w^{-\nu}\right), \quad \text { as } w \rightarrow+\infty .
$$

In the case of Orlicz spaces, for every $f \in L^{\varphi}\left(\mathbb{R}^{n}\right)$, the following holds:

$$
I^{\varphi}\left[\lambda S_{w}^{\mathcal{F}_{n}} f\right] \leq \frac{1}{\delta^{n}} I^{\varphi}[\lambda f],
$$

for some $\lambda>0$ and for every $w>0$, since $\left\|\mathcal{F}_{n}\right\|_{1}=1$ and $m_{0, \Pi^{n}}\left(\mathcal{F}_{n}\right)=$ 1. In particular, $S_{w}^{\mathcal{F}_{n}} f \in L^{\varphi}\left(\mathbb{R}^{n}\right)$ whenever $f \in L^{\varphi}\left(\mathbb{R}^{n}\right)$. 
Moreover, for any $f \in L^{\varphi}\left(\mathbb{R}^{n}\right) \cap \operatorname{Lip}_{\varphi}(\nu), 0<\nu \leq 1$, there exists a $\lambda>0$ such that

$$
I^{\varphi}\left[\lambda\left(S_{w}^{\mathcal{F}_{n}} f-f\right)\right]=\mathcal{O}\left(w^{-\nu}\right), \quad \text { as } w \rightarrow+\infty .
$$

The Fejér's kernel $\mathcal{F}_{n}$ provides an example of a kernel with unbounded support. In this case, for practical applications of the above reconstruction formula to any given signal with unbounded duration, one should evaluate the sampling series $S_{w} f$ at any fixed $\underline{x} \in \mathbb{R}^{n}$, and this requires one to know an infinite number of mean values $w^{n} \int_{R_{k}^{w}} f(\underline{u}) d \underline{u}$. Clearly, in order to evaluate the operators $S_{w} f$ at $\underline{x}$, the infinite sampling series must be truncated to a finite one, and this procedure leads to the so-called truncation error. However, if the signal $f$ has bounded duration, i.e., compact support, this problem does not arise.

In order to avoid the truncation error, kernels $\chi$ with compact support can be taken into consideration.

Noteworthy examples of such kernels can be constructed using the well-known, univariate central B-spline of order $k \in \mathbb{N}$, defined by

$$
M_{k}(x):=\frac{1}{(k-1) !} \sum_{i=0}^{k}(-1)^{i}\left(\begin{array}{l}
k \\
i
\end{array}\right)\left(\frac{k}{2}+x-i\right)_{+}^{k-1} .
$$

We recall that $(x)_{+}:=\max \{x, 0\}$ denotes the positive part of $x \in \mathbb{R}$ (see $[3,27,48])$. We have that the Fourier transform of $M_{k}$ is given by

$$
\widehat{M_{k}}(v):=\operatorname{sinc}^{n}\left(\frac{v}{2 \pi}\right), \quad(v \in \mathbb{R}),
$$

and then, if we consider the case of the uniformly spaced sampling scheme, condition (10) is satisfied for every $\mu>0$ by Remark 6.1. Clearly, $M_{k}$ are bounded on $\mathbb{R}$, with compact support [ $\left.-k / 2, k / 2\right]$, and hence $M_{k} \in L^{1}(\mathbb{R})$, for all $k \in \mathbb{N}$. Moreover, it is easy to deduce that conditions (9) and (11) are fulfilled for every $\beta>0$ and $\alpha>0$. As in the case of Fejér's kernel, we define the multivariate B-spline kernel of order $k \in \mathbb{N}^{+}$by

$$
\mathcal{M}_{k}^{n}(\underline{x}):=\prod_{i=1}^{n} M_{k}\left(x_{i}\right), \quad \underline{x}=\left(x_{1}, \ldots, x_{n}\right) \in \mathbb{R}^{n} .
$$


Then the multivariate sampling Kantorovich operators based on the $\mathrm{B}$-spline kernel of order $k$, in the case of the uniform sampling scheme, now take the form

$$
\left(S_{w}^{\mathcal{M}_{k}^{n}} f\right)(x)=\sum_{\underline{k} \in \mathbb{Z}^{n}}\left[w^{n} \int_{R_{\underline{k}}^{w}} f(\underline{u}) d \underline{u}\right] \mathcal{M}_{k}^{n}(w \underline{x}-\underline{k}), \quad\left(\underline{x} \in \mathbb{R}^{n}\right),
$$

for every $w>0$, where $f: \mathbb{R}^{n} \rightarrow \mathbb{R}$ is a locally integrable function such that the above series is convergent for every $\underline{x} \in \mathbb{R}^{n}$. From Theorem 4.1, Theorem 5.1 and Corollary 5.3, we obtain the following:

Corollary 6.3. Let $f \in \operatorname{Lip}_{\infty}(\nu)$, with $0<\nu \leq 1$. Then

$$
\left\|S_{w}^{\mathcal{M}_{k}^{n}} f-f\right\|_{\infty}=\mathcal{O}\left(w^{-\nu}\right), \quad \text { as } \quad w \rightarrow+\infty .
$$

In the case of Orlicz spaces, for every $f \in L^{\varphi}\left(\mathbb{R}^{n}\right)$, the following holds:

$$
I^{\varphi}\left[\lambda S_{w}^{\mathcal{M}_{k}^{n}} f\right] \leq \frac{1}{\delta^{n}} I^{\varphi}[\lambda f]
$$

for some $\lambda>0$ and for every $w>0$. In particular, $S_{w}^{\mathcal{M}_{k}^{n}} f \in L^{\varphi}\left(\mathbb{R}^{n}\right)$ whenever $f \in L^{\varphi}\left(\mathbb{R}^{n}\right)$.

Moreover, for any $f \in L^{\varphi}\left(\mathbb{R}^{n}\right) \cap \operatorname{Lip}_{\varphi}(\nu), 0<\nu \leq 1$, there exists a $\lambda>0$ such that

$$
I^{\varphi}\left[\lambda\left(S_{w}^{\mathcal{M}_{k}^{n}} f-f\right)\right]=\mathcal{O}\left(w^{-\nu}\right), \quad \text { as } \quad w \rightarrow+\infty .
$$

For other useful examples of kernels, see, e.g., $[\mathbf{3}, \mathbf{7}, \mathbf{1 3}, \mathbf{4 7}, \mathbf{1 7}$, $23,24,22,20,21,25]$.

Acknowledgments. The authors are members of the Gruppo Nazionale per l'Analisi Matematica, la Probabilitá e le loro Applicazioni (GNAMPA) of the Istituto Nazionale di Alta Matematica (INdAM).

\section{REFERENCES}

1. L. Angeloni and G. Vinti, Rate of approximation for nonlinear integral operators with applications to signal processing, Diff. Int. Eq. 18 (2005), 855-890.

2. C. Bardaro, P.L. Butzer, R.L. Stens and G. Vinti, Approximation of the Whittaker sampling series in terms of an average modulus of smoothness covering discontinuous signals, J. Math. Anal. Appl. 316 (2006), 269-306. 
3. C. Bardaro, P.L. Butzer, R.L. Stens and G. Vinti, Kantorovich-type generalized sampling series in the setting of Orlicz spaces, Sampl. Theor. Signal Image Process. 6 (2007), 29-52.

4. , Prediction by samples from the past with error estimates covering discontinuous signals, IEEE Trans. Inform. Theor. 56 (2010), 614-633.

5. C. Bardaro and I. Mantellini, Modular approximation by sequences of nonlinear integral operators in Musielak-Orlicz spaces, Atti Sem. Mat. Fis. Univ. Modena 46 (1998), 403-425.

6. , On convergence properties for a class of Kantorovich discrete operators, Numer. Funct. Anal. Optim. 33 (2012), 374-396.

7. C. Bardaro, J. Musielak and G. Vinti, Nonlinear integral operators and applications, De Gruyter Ser. Nonlin. Anal. Appl. 9 (2003), New York.

8. C. Bardaro and G. Vinti, Some inclusion theorems for Orlicz and MusielakOrlicz type spaces, Ann. Mat. Pura Appl. 168 (1995), 189-203.

9. A general approach to the convergence theorems of generalized sampling series, Appl. Anal. 64 (1997), 203-217.

10. An abstract approach to sampling type operators inspired by the work of P.L. Butzer-Part I-Linear operators, Sampl. Theor. Sig. Image Process. 2 (2003), 271-296.

11. L. Bezuglaya and V. Katsnelson, The sampling theorem for functions with limited multi-band spectrum I, Z. Anal. Anwend. 12 (1993), 511-534.

12. P.L. Butzer, A. Fisher and R.L. Stens, Generalized sampling approximation of multivariate signals: theory and applications, Note Matem. 10 (1990), 173-191.

13. P.L. Butzer and R.J. Nessel, Fourier analysis and approximation I, Academic Press, New York, 1971.

14. P.L. Butzer, S. Ries and R.L. Stens, Approximation of continuous and discontinuous functions by generalized sampling series, J. Approx. Theor. 50 (1987), 25-39.

15. P.L. Butzer and R.L. Stens, Sampling theory for not necessarily band-limited functions: a historical overview, SIAM Rev. 34 (1992), 40-53.

16. , Linear prediction by samples from the past, in Advanced topics in Shannon sampling and interpolation theory, R.J. Marks II, eds., Springer-Verlag, New York, 1993.

17. F. Cluni, D. Costarelli, A.M. Minotti and G. Vinti, Multivariate sampling Kantorovich operators: approximation and applications to civil engineering, EURASIP Open Library, Proc. SampTA 2013, 10th International Conference on Sampling Theory and Applications, July 1-5, 2013, Jacobs University, Bremen, 2013, 400-403.

18. _ Enhancement of thermographic images as tool for structural analysis in earthquake engineering, NDT\&E Inter. Indep. Nondestr. Test. Eval. (2014) DOI: $10.1016 / \mathrm{j}$.ndteint.2014.10.001.

19. - Applications of sampling Kantorovich operators to thermographic images for seismic engineering, J. Comp. Anal. Appl. 19 (2015), 602-617. 
20. D. Costarelli, Interpolation by neural network operators activated by ramp functions, J. Math. Anal. Appl. 419 (2014), 574-582.

21. , Neural network operators: constructive interpolation of multivariate functions, submitted, 2014.

22. D. Costarelli and R. Spigler, Approximation by series of sigmoidal functions with applications to neural networks, Ann. Matem. Pura Appl., 2013, doi: 10.1007/s10231-013-0378-y.

23. Approximation results for neural network operators activated by sigmoidal functions, Neural Networks 44 (2013), 101-106.

24. Multivariate neural network operators with sigmoidal activation functions, Neural Networks 48 (2013), 72-77.

25. Convergence of a family of neural network operators of the Kantorovich type, J. Approx. Theor. 185 (2014), 80-90.

26. , How sharp is Jensen's inequality?, submitted.

27. D. Costarelli and G. Vinti, Approximation by multivariate generalized sampling Kantorovich operators in the setting of Orlicz spaces, Boll. U.M.I. 4 (2011), $445-468$.

28. Approximation by nonlinear multivariate sampling Kantorovich type operators and applications to image processing, Num. Funct. Anal. Opt. 34 (2013), 819-844.

29. , Order of approximation for sampling Kantorovich operators, J. Int. Equat. Appl. 26 (2014), 345-368.

30. , Order of approximation for nonlinear sampling Kantorovich operators in Orlicz spaces, Comment. Math. 5 (2013), 171-192.

31. M.M. Dodson and A.M. Silva, Fourier analysis and the sampling theorem, Proc. Ir. Acad. 86 (1985), 81-108.

32. C. Donnini and G. Vinti, Approximation by means of Kantorovich generalized sampling operators in Musielak-Orlicz spaces, PanAmer. Math. J. 18 (2008), $1-18$.

33. J.R. Higgins, Sampling theory in Fourier and signal analysis: Foundations, Oxford Univ. Press, Oxford, 1996.

34. J.R. Higgins and R.L. Stens, Sampling theory in Fourier and signal analysis: Advanced topics, Oxford Science Publications, Oxford University Press, Oxford, 1999.

35. A.J. Jerry, The Shannon sampling-its various extensions and applications: a tutorial review, Proc. IEEE 65 (1977), 1565-1596.

36. W.M. Kozlowski, Modular function spaces, Pure Appl. Math., Marcel Dekker, New York, 1988.

37. M.A. Krasnosel'skii and Ya.B. Rutickii, Convex functions and Orlicz spaces, P. Noordhoff Ltd., Groningen, The Netherlands, 1961.

38. L. Maligranda, Orlicz spaces and interpolation, Sem. Mat. IMECC, Campinas, 1989. 
39. J. Musielak, Orlicz spaces and modular spaces, Lect. Notes Math. 1034, Springer-Verlag, New York, 1983.

40. J. Musielak and W. Orlicz, On modular spaces, Stud. Math. 28 (1959), $49-65$.

41. M.M. Rao and Z.D. Ren, Theory of Orlicz spaces, Pure Appl. Math., Marcel Dekker, Inc., New York, 1991.

42. Applications of Orlicz spaces, Mono. Text. Pure Appl. Math. 250, Marcel Dekker, Inc., New York, 2002.

43. S. Ries and R.L. Stens, Approximation by generalized sampling series, in Constructive theory of functions'84, Sofia, 1984, 746-756.

44. F. Ventriglia and G. Vinti, A unified approach for the convergence of nonlinear Kantorovich type operators, Comm. Appl. Nonlin. Anal. 21 (2014), 4574 .

45. C. Vinti, A survey on recent results of the mathematical seminar in Perugia, inspired by the Work of Professor P.L. Butzer, Result. Math. 34 (1998), 32-55.

46. G. Vinti, A general approximation result for nonlinear integral operators and applications to signal processing, Appl. Anal. 79 (2001), 217-238.

47. , Approximation in Orlicz spaces for linear integral operators and applications, Rend. Circ. Mat. Palermo 76 (2005), 103-127.

48. G. Vinti and L. Zampogni, Approximation by means of nonlinear Kantorovich sampling type operators in Orlicz spaces, J. Approx. Theor. 161 (2009), $511-528$.

49. A Anifying approach to convergence of linear sampling type operators in Orlicz spaces, Adv. Diff. Equat. 16 (2011), 573-600.

50. _ Approximation results for a general class of Kantorovich type operators, Adv. Nonlin. Stud. 14 (2014), 991-1011.

Dipartimento di Matematica e Informatica, Università degli Studi di PeRugia, 1, Via Vanvitelli, 06123, Perugia, Italy

Email address: danilo.costarelli@gmail.com

Dipartimento di Matematica e Informatica, Università degli Studi di Perugia, 1, Via Vanvitelli, 06123, Perugia, Italy

Email address: gianluca.vinti@unipg.it 